\title{
Numerical replica limit for the density correlation of the random Dirac fermion
}

\author{
Shinsei Ryu ${ }^{1}$ and Yasuhiro Hatsugai ${ }^{1,2}$ \\ ${ }^{1}$ Department of Applied Physics, University of Tokyo, 7-3-1 Hongo Bunkyo-ku, Tokyo 113-8656, Japan \\ ${ }^{2}$ PRESTO, JST, Saitama 332-0012, Japan
}

(Received 2 February 2001; published 31 May 2001)

\begin{abstract}
The zero-mode wave function of a massless Dirac fermion in the presence of a random gauge field is studied. The density correlation function is calculated numerically, and found to exhibit a power law in the weak randomness with a disorder-dependent exponent. It deviates from the power law, and the disorder dependence becomes frozen in the strong randomness. A classical statistical system is employed through the replica trick to interpret the results, and the direct evaluation of the replica limit is demonstrated numerically. Analytical expressions of the correlation function and the free energy are also discussed with the replica symmetry breaking and the Liouville field theory.
\end{abstract}

DOI: 10.1103/PhysRevB.63.233307

PACS number(s): 72.15.Rn, 75.10.Nr

Although the scaling theory of localization for twodimensional (2D) disordered systems generally predicts the absence of extended states, we have some examples of nonlocalized states in two dimensions which are marginally allowed to appear. Among these, systems with chiral symmetry such as Gade's model, ${ }^{1}$ the random flux model, ${ }^{2}$ or the $\pi$-flux model with link disorders ${ }^{3}$ have attracted much attention. Compared to the conventional models, where randomness enters as a on-site potential, randomness resides on links (i.e., as a gauge field) in these models. This type of randomness induces a special symmetry which affects the localization properties drastically. Due to this symmetry, the densities of states of the models have singularities at zero energy, and the corresponding wave functions exhibit a multifractal behavior.

Now let us concentrate our attention on cases where the low-lying physics is described by the Dirac fermions. Examples of these include the $\pi$-flux model mentioned above, the Chalker-Coddington network model, ${ }^{4}$ and the gap closing (quantum Hall) transition. ${ }^{5}$ 2D $d$-wave superconductivity is another important example. The question of how disorder affects the low-energy excitations, and whether all states are localized, have been under extensive study recently. ${ }^{6}$ Focusing on one node, let us consider a Hamiltonian of the form $H=\boldsymbol{\sigma} \cdot \mathbf{p}+\boldsymbol{\sigma} \cdot \mathbf{A}$ where $\sigma_{i=x, y}$ are the usual $2 \times 2$ Pauli matrices and $\mathbf{A}$ is a random gauge field. This Hamiltonian has a continuum version of the chiral symmetry $\{H, \gamma\}=0$, where $\gamma=\sigma_{z}$. Remarkably, in this situation, an explicit construction of zero energy wave functions is possible in a $2 \mathrm{D}$ continuum space. ${ }^{7,8}$ The Schrödinger (Dirac) equations for the zero modes are

$$
(2 \partial+i \bar{A}) \psi_{-}=0, \quad(2 \bar{\partial}+i A) \psi_{+}=0,
$$

where $\partial:=\left(\partial_{x}-i \partial_{y}\right) / 2, \quad \bar{\partial}:=\left(\partial_{x}+i \partial_{y}\right) / 2, A:=A_{x}+i A_{y}$, and $\bar{A}:=A_{x}-i A_{y} .{ }^{9}$ If we adopt a Coulomb gauge to express the vector potential $\mathbf{A}$ in terms of a scalar potential $\Phi$ as $A_{x}$ $=\partial_{y} \Phi$ and $A_{y}=-\partial_{x} \Phi$, and assume that the mean total flux piercing the system is zero, we can obtain an exact solution for any realization of disorders as $\psi_{ \pm}(\mathbf{x})=C_{ \pm} e^{\mp \Phi(\mathbf{x})}$. Further, let us assume that the probability weight for each realization of $\Phi(\mathbf{x})$ has the form $P[\Phi] \propto e^{-S}$ where $S[\Phi]$
$=1 / 2 g \int d^{2} x[\nabla \Phi(\mathbf{x})]^{2}$ and $g$ is the disorder strength or, in field-theoretic language, the coupling constant, which is $d i$ mensionless in two dimensions.

From now on, we concentrate our attention only on $\psi_{+}$. For physical interest, it is necessary to consider normalized wave functions in an $L \times L$ box as $\psi(\mathbf{x})=e^{-\Phi(\mathbf{x})} / \sqrt{Z}$ with $Z=\int\left(d^{2} \mathbf{x} / a^{2}\right) e^{-2 \Phi(\mathbf{x})}$, where $a$ is a lattice constant. Here we regularized the problem on an $N \times N$ periodic lattice, although the original problem is formulated in a continuum space (where $L=N a$ ). Correspondingly, we use the probability weight $S[\Phi] \propto 1 / 2 g \Sigma_{\langle i j\rangle}\left(\Phi_{i}-\Phi_{j}\right)^{2}$ or, in momentum space, $S[\Phi] \propto N^{2} / g \Sigma_{m} \tilde{\Phi}_{m} \tilde{\Phi}_{-m}\left[2-\Sigma_{\mu=1}^{2} \cos \left(a k_{m}^{\mu}\right)\right]$, where $\widetilde{\Phi}_{m}:=N^{-2} \sum_{j} e^{-i k_{m} x_{j}} \Phi_{j}$ and the sum extends over the first Brillouin zone $m^{\mu}=-N / 2+1 \cdots N / 2$ with $k_{m}^{\mu}=2 \pi m^{\mu} / a N$ ( $N$ is even for convenience.). We put $\widetilde{\Phi}_{m=0}=0$. Typical probability densities $|\psi(x)|^{2}$ calculated numerically are shown in Fig. 1, which reminds us of multifractal states found at a localization-delocalization transition for several systems. ${ }^{10}$

In fact, the multifractal property of this wave function has been revealed quantitatively by a close analogy to a generalized random energy model. ${ }^{11,12}$ As the disorder strength $g$ is varied, the multifractal spectrum exhibits a sharp transition which is similar to the freezing phenomenon in spin glasses. Several other approaches such as the supersymmetry (SUSY) technique ${ }^{13}$ the connection to the Liouville field theory, ${ }^{14}$ the renormalization group, ${ }^{15}$ or conformal field theory ${ }^{16}$ have also been taken to support the transition.

Since the calculated probability densities (Fig. 1) are so spiky, the discretization procedure above may not be justified. In spite of this subtlety, we concentrate on this welldefined discretized wave function to investigate universal properties.

In this paper, we evaluate the density correlation function

$$
\left\langle\psi^{2}(1) \psi^{2}(2)\right\rangle=\left\langle\frac{1}{Z^{2}} e^{-2 \Phi\left(\mathbf{x}_{1}\right)} e^{-2 \Phi\left(\mathbf{x}_{2}\right)}\right\rangle,
$$

where $\langle\cdots\rangle$ denotes the averaging with respect to the weight $P[\Phi]$. Here the difficulties reside in the normalization factor 

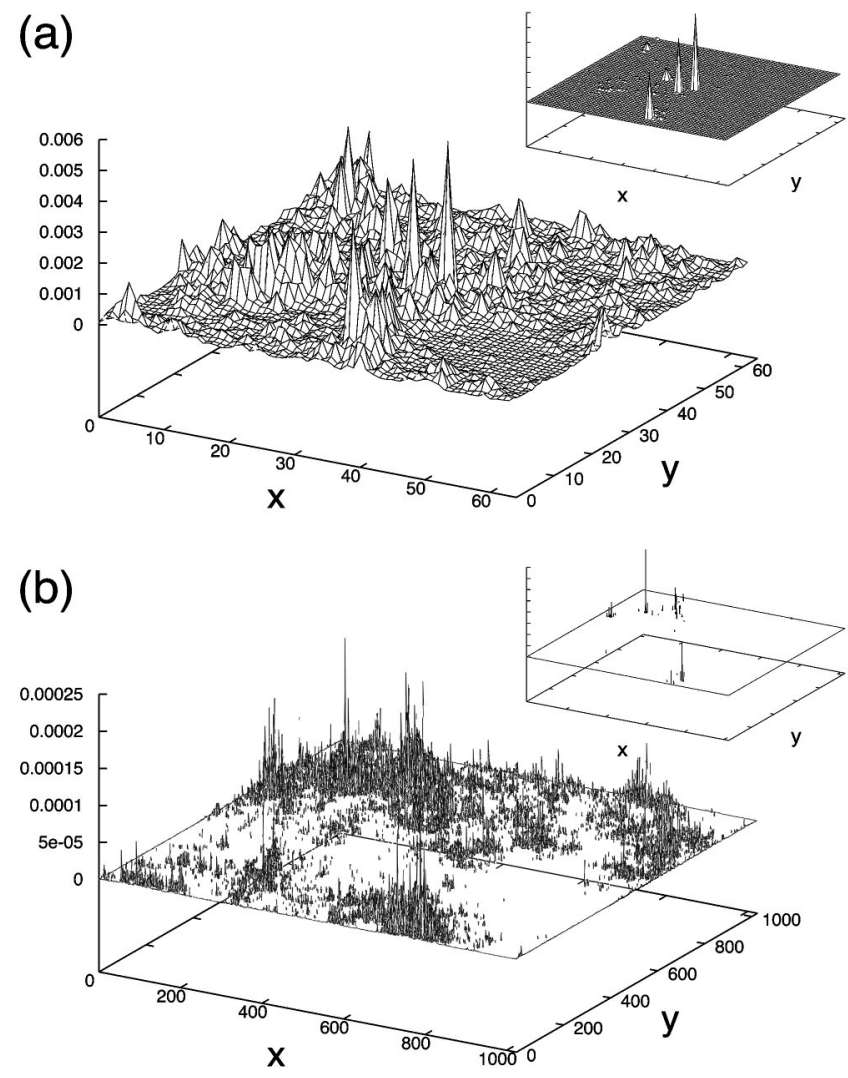

FIG. 1. Typical probability densities $|\psi(x)|^{2}$ for $g=0.4$ on (a) a $64 \times 64$ lattice and (b) a $1024 \times 1024$ lattice. Insets: $|\psi(x)|^{2}$ for $g$ $=6.4$ for each system.

$Z$ in the denominator, since $Z$ itself is a random variable. One of the simplest attempts to cope with this is the replica trick. We multiply the numerator by $Z^{n}$, and consider

$$
\begin{aligned}
\left\langle\psi^{2}(1) \psi^{2}(2)\right\rangle_{n}:= & \int \frac{d^{2} \xi_{1}}{a^{2}} \cdots \frac{d^{2} \xi_{n-2}}{a^{2}} \\
& \times\left\langle e^{-2\left[\Phi\left(\mathbf{x}_{1}\right)+\Phi\left(\mathbf{x}_{2}\right)+\Phi\left(\xi_{1}\right)+\cdots+\Phi\left(\xi_{n-2}\right)\right]}\right\rangle,
\end{aligned}
$$

which is expected to reduce to $\left\langle\psi^{2}(1) \psi^{2}(2)\right\rangle$ by taking the replica limit $n \rightarrow 0$ (analytical continuation). We use this replica trick to interpret the direct numerical results, and also try to take the replica limit by evaluating $\left\langle\psi^{2}(1) \psi^{2}(2)\right\rangle_{n}$ numerically for several $n$ and extrapolating them to $n=0$. In addition, we utilize the evaluation of $\left\langle\psi^{2}(1) \psi^{2}(2)\right\rangle_{n}$, together with the Liouville field theory, to obtain an analytical expression of the correlation function for the weak disorder regime.

First let us present a direct numerical calculation of $\left\langle\psi^{2}(1) \psi^{2}(2)\right\rangle$. In this problem, the probability weight itself is diagonal in momentum space (see above), which allows us to carry out numerical simulations with a very large lattice up to $2048 \times 2048$. Figure 2 shows calculated $\left\langle\psi^{2}(1) \psi^{2}(2)\right\rangle$ for various $g$ on a $1024 \times 1024$ lattice. The quenched averaging is performed over $\sim 10^{5}$ different realization of disorders. As shown, the correlation function for the weak disor-
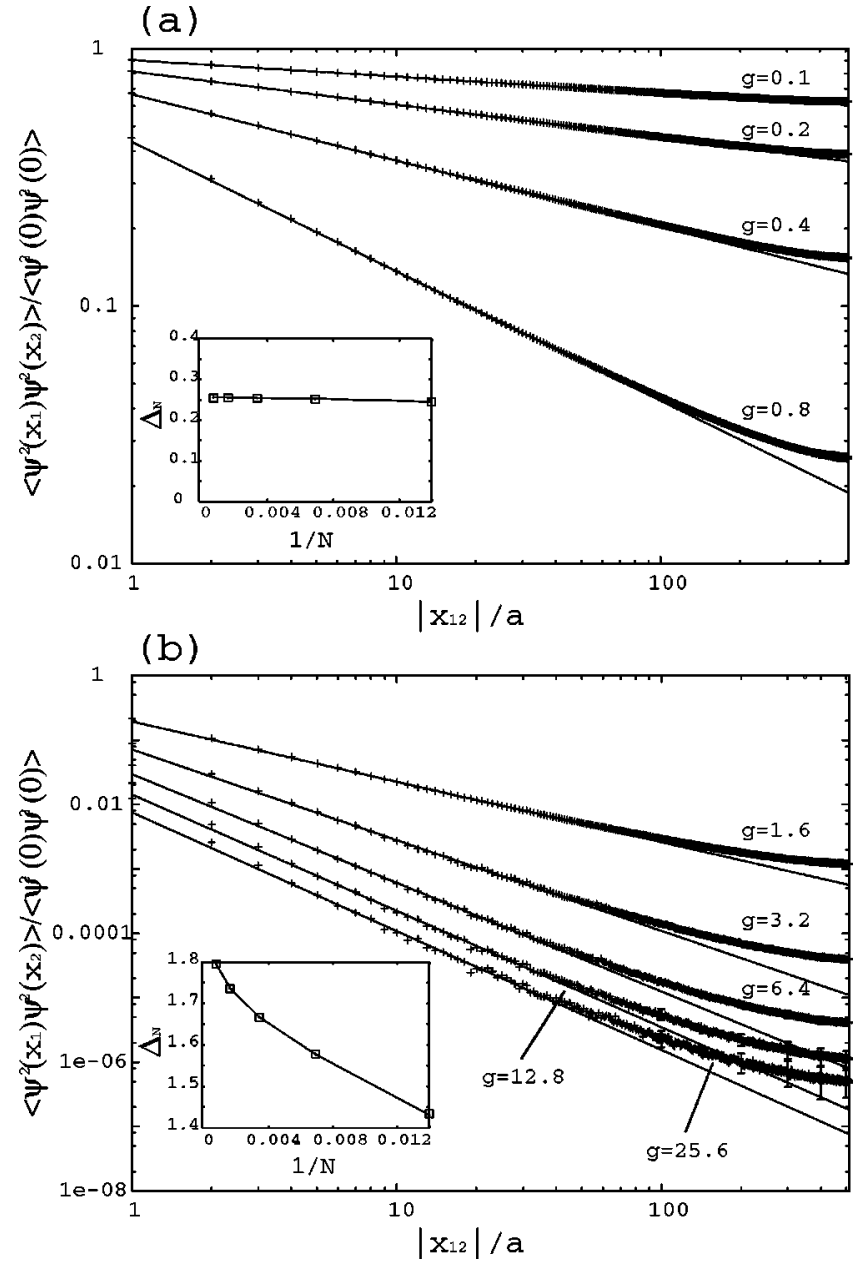

FIG. 2. The numerically calculated density correlation for (a) weak and (b) strong disorder regimes on a $1024 \times 1024$ lattice. For $g \leqslant 1.6$, the statistical error is smaller than the data point. For $g$ $\geqslant 3.2$, the error bars are shown for every 100 points. Insets: the "exponent" $\Delta_{N}$ vs $1 / N$ for (a) $g=0.4$ and (b) $g=6.4$. It is evaluated for $1 \leqslant\left|x_{12}\right| / a \leqslant 10$ for each finite system.

der exhibits a power-law behavior $\left\langle\psi^{2}(1) \psi^{2}(2)\right\rangle \sim\left|x_{12}\right|^{-\Delta}$ for $1 \lesssim\left|x_{12}\right| / a \ll N / 2$, with its exponent $\Delta$ dependent linearly on $g, \Delta=2 g / \pi$ (Fig. 3). This is consistent with several analytical approaches. ${ }^{3}$ As $g$ increases, however, the $g$ dependence of the correlation function becomes weaker, and it deviates from the power law. To be more precise, there is a systematic deviation from the simple power law; that is, if we determine the "exponent" $\Delta_{N}$ on a finite $N$ system, it seems to diverge as $N$ increases (see the insets of Fig. 2). This is clearly different from the behavior of $\Delta_{N}$ in the weak randomness where $\Delta_{N}$ seems to converge. In fact, as shown in Fig. 1, the wave function becomes peaked on a few sites as $g$ increases. However, it is different from the usual localized wave function which decays exponentially with its typical length scale characterized by the localization length. The above change of behavior in the correlation function is consistent with the transition from weak to strong disorder found in the multifractal spectrum in the previous studies.

Next let us try to interpret the above numerical results by the replica trick. After replicating $Z$, we can perform the averaging $\langle\cdots\rangle$ in Eq. (2), and obtain 


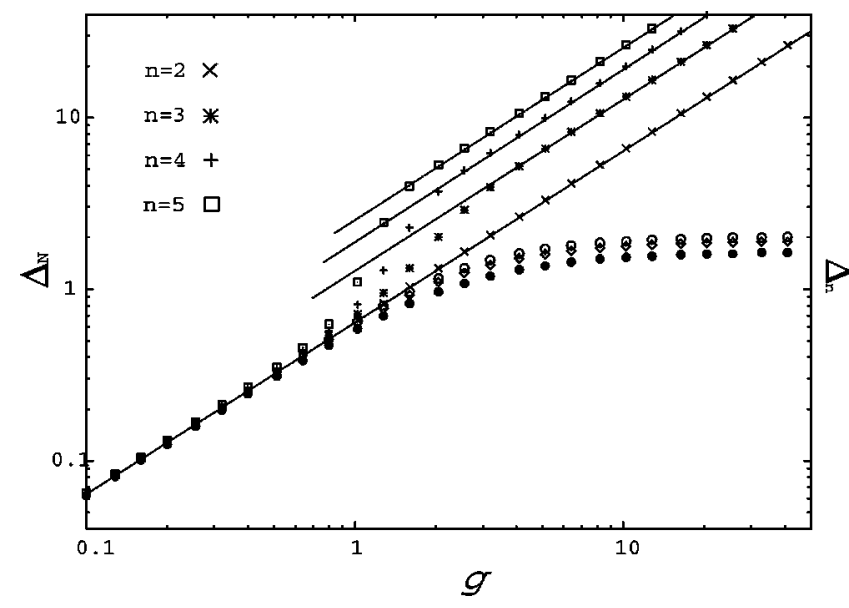

FIG. 3. The "exponent" $\Delta_{N}$ with respect to $g$ and its naive replica estimates $\Delta_{n=2,3,4,5} \cdot \Delta_{N}$ is evaluated for $1 \leqslant\left|x_{12}\right| / a \leqslant 10$ for $64 \times 64(\bigcirc), 256 \times 256(\diamond)$, and $1024 \times 1024(\bullet)$ systems. The statistical error is smaller than the symbol size. The analytical estimation for $\Delta_{n}$ is represented by lines.

$$
\left\langle\psi^{2}(1) \psi^{2}(2)\right\rangle_{n}=\left\langle\delta\left(\xi_{k}-x_{1}\right) \delta\left(\xi_{l}-x_{2}\right)\right\rangle_{n}^{c l}=: \Gamma_{k, l}^{n}(1,2)
$$

with

$$
\langle O\rangle_{n}^{c l}:=\operatorname{Tr}\left[O e^{-H_{n}}\right] / \operatorname{Tr}\left[e^{-H_{n}}\right],
$$

where

$$
\operatorname{Tr}:=\frac{1}{n !} \int \frac{d^{2} \xi_{1}}{a^{2}} \cdots \frac{d^{2} \xi_{n}}{a^{2}}
$$

$-H_{n}:=4 \sum_{k<l}^{n} \mathcal{G}\left(\xi_{k}, \xi_{l}\right), \quad G\left(x_{i}, x_{j}\right):=\left\langle\Phi\left(x_{i}\right) \Phi\left(x_{j}\right)\right\rangle$ is the Green's function, and $\mathcal{G}\left(x_{i}, x_{j}\right):=G\left(x_{i}, x_{j}\right)-G(0) \sim$ $-g / 2 \pi \ln \left(\left|x_{i j}\right| / a\right)$. Here we multiply some trivial factors which reduce to unity in the limit $n \rightarrow 0$. As suggested in Eq. (3), $\left\langle\psi^{2}(1) \psi^{2}(2)\right\rangle_{n}$ can be interpreted as the two-body density of a classical statistical system consisting of a set of particles (replicas) interacting with each other via the potential $\mathcal{G}\left(x_{i}, x_{j}\right)$. These replica estimations are shown and directly compared to the numerical results (Fig. 4). In Fig. 4, $\left\langle\psi^{2}(1) \psi^{2}(2)\right\rangle_{n}$ for various $n$ (the number of replicas) with fixed $g$ are obtained by calculating Eq. (3) numerically on a $64 \times 64$ lattice. This rather small lattice size is due to the multiple integral in Eq. (3). Note that we do not have $\left\langle\psi^{2}(1) \psi^{2}(2)\right\rangle_{n=1}$, as inferred from Eq. (2). For $g=0.4$, the replica estimation seems to converge to the one calculated by the direct numerical simulations. For $g=6.4$, in contrast, it hardly seems to coincide with the exact one in the limit $n$ $\rightarrow 0$. Moreover, it gives an unphysical result, i.e., a negative exponent, after taking the replica limit.

This breakdown is closely related to the transition in the replica space. There are two distinct phases for this system. For small $g$, all configurations are equally favorable. As $g$ increases, however, the configurations where all replicas are close to each other come to have a large weight. Thus, for sufficiently small $g$, it is enough to concentrate on only $\xi_{k}$ and $\xi_{l}$ in Eq. (3). The configuration of the other particles is
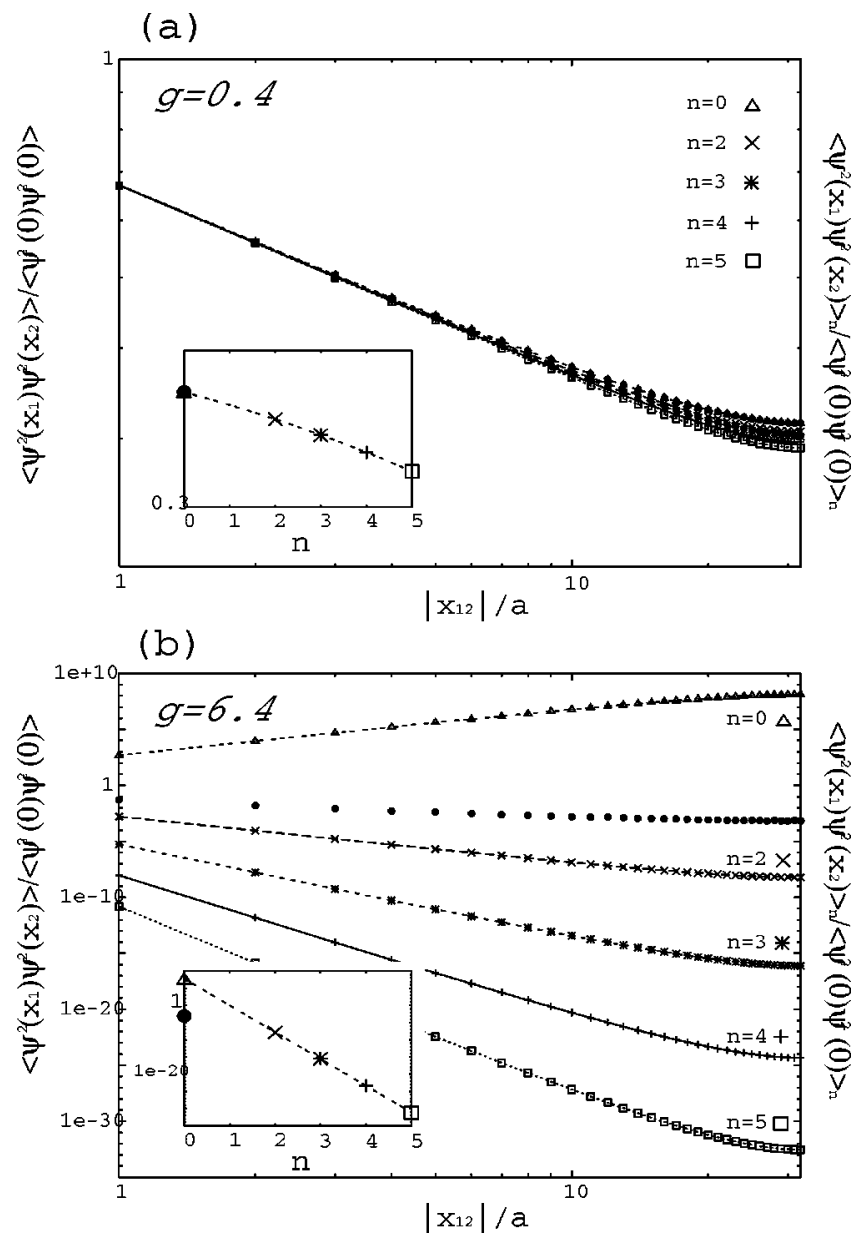

FIG. 4. The numerically calculated density correlations $(0)$ and their replica estimates with $n=2,3,4$, and 5 for (a) $g=0.4$ and (b) $g=6.4$ on a $64 \times 64$ periodic lattice. The replica estimates are obtained by evaluating the multiple integral in Eq. (3) directly. $\left\langle\psi^{2}\left(x_{1}\right) \psi^{2}\left(x_{2}\right)\right\rangle_{n=0}$ is obtained by extrapolating from $\left\langle\psi^{2}\left(x_{1}\right) \psi^{2}\left(x_{2}\right)\right\rangle_{n=2,3,4,5}$. Insets: $\left\langle\psi^{2}\left(x_{1}\right) \psi^{2}\left(x_{2}\right)\right\rangle_{n}$ vs $n$ at $\left|x_{12}\right| / a$ $=20$ (semilog plot).

smeared out in the ensemble average, and irrelevant for $\left\langle\psi^{2}(1) \psi^{2}(2)\right\rangle_{n}$. For large $g$, on the other hand, the main contributions in the ensemble average are from the configurations where all replicas except $\xi_{l}$ (or $\xi_{k}$ ) are at $x_{1}$ (or $x_{2}$, respectively). Then $\left\langle\psi^{2}(1) \psi^{2}(2)\right\rangle_{n}$ is expected to behave as

$$
\left\langle\psi^{2}(1) \psi^{2}(2)\right\rangle_{n} \sim 1 /\left|x_{12}\right|^{\Delta_{n}},
$$

with $\Delta_{n} \sim 2 g / \pi$ for small $g$ and $\sim 2 g(n-1) / \pi$ for large $g$. A numerically calculated $\Delta_{n}$ is shown in Fig. 3, which confirms that the above estimation for small $n$ is reasonable. Taking the replica limit $n \rightarrow 0$, we obtain $\Delta_{n=0}=2 g / \pi$ for small $g$, which is consistent with the results obtained by the SUSY technique. ${ }^{13}$ For the strong disorder regime, however, the exponent reduces to $-2 g / \pi$, which is unphysical. Does this mean that the replica trick is a mere trick? However, the replica symmetry-breaking (RSB) solution, which was proposed for the free energy, ${ }^{15}$ may also be applicable for the correlation function, and give the correct answer both for weak and strong disorder. 
We also investigated other types of correlation functions such as $\langle\psi(1) \psi(2)\rangle$ or $\left\langle\Phi(1) \psi^{2}(1) \Phi(2) \psi^{2}(2)\right\rangle$, the latter of which is of interest because it is related to the second derivative of the free energy $\langle\ln Z\rangle / \ln (L / a)$, which shows the nonanalyticity at $g=2 \pi$. Their behaviors are qualitatively similar to that of $\left\langle\psi^{2}(1) \psi^{2}(2)\right\rangle$ in that, for small $g$, these correlation functions become steeper and steeper as $g$ increases; their $g$ dependences are calculable by the replica trick. For large $g$, however, their $g$ dependencies are rather weak, and the naive replica trick fails.

Another interesting approach is to utilize the formula $1 / Z^{N}=[1 /(N-1) !] \int_{0}^{\infty} d \mu e^{-\mu Z} \mu^{N-1}$, and express the correlation function as $\left\langle\psi^{2}(1) \psi^{2}(2)\right\rangle$ $=\int_{0}^{\infty} d \mu \mu \int \mathcal{D} \Phi e^{-2 \Phi\left(x_{1}\right)-2 \Phi\left(x_{2}\right)} e^{-S_{L F T}[\Phi]} \quad$ where $\quad S_{L F T}$ $=\int\left(d^{2} \xi / a^{2}\right)\left[1 / 2 g(\nabla \Phi)^{2}+\mu e^{-2 \Phi(\xi)}\right] .{ }^{14}$ This action resembles that of the Liouville field theory in 2D quantum gravity. However, since it was pointed out that there are some subtleties about the field theoretic treatment, ${ }^{15}$ we evaluate it directly by using the replica estimates. We expand $e^{-\mu Z}$ to express $\left\langle\psi^{2}(1) \psi^{2}(2)\right\rangle$ as a superposition of the replica estimates with a different number of replicas, i.e., the grand canonical ensemble

$$
\left\langle\psi^{2}(1) \psi^{2}(2)\right\rangle=2 \int_{-\infty}^{\infty} d \tilde{\mu} \sum_{n=2}^{\infty}(-1)^{n} e^{-\mathcal{F}_{n}(\tilde{\mu})} \sum_{k \neq l}^{n} \Gamma_{k, l}^{n}(1,2),
$$

where $\mathcal{F}_{n}(\tilde{\mu})=-\ln \operatorname{Tr} e^{-\left(H_{n}-\tilde{\mu} n\right)}+2 n^{2} G(0)$ and $\mu=e^{\tilde{\mu}}$. Since, for weak disorder, the summand depends only trivially on $n$, we can easily sum up this suggestive expression and obtain the same result as the replica trick.
This method is also applicable for the free energy. We expand $\ln Z=\int_{0}^{\infty}(d \mu / \mu)\left(e^{-\mu}-e^{-\mu Z}\right)$ as

$$
\langle\ln Z\rangle=\int_{-\infty}^{\infty} d \tilde{\mu}\left(e^{-e^{\tilde{\mu}}}-1-\sum_{n=1}^{\infty}(-1)^{n} e^{-} \mathcal{F}_{n}(\tilde{\mu})\right)
$$

It is difficult to perform the summation for strong disorder, though we can obtain the correct answer for weak disorder. However, if we simply employ the RSB estimate of Carpentier and Doussal, ${ }^{15} e^{-} \mathcal{F}_{n}=n !^{-1}(L / a)^{(p g / \pi+2 / p+\tilde{\mu}) n}$, where $p=1$ for the weak disorder and $\sqrt{2 \pi / g}$ for the strong disorder, the summation reproduces the exact result both for the weak and strong disorder regimes. ${ }^{17}$

In conclusion, we have numerically investigated the density correlation of the random Dirac fermion at zero energy, which exhibits a drastic change of behavior with respect to the randomness. However, the peculiar nature of the strong disorder phase is still unclear and further investigations by several approaches (RSB, etc.) are needed. Another interesting issue is whether the transition affects nonzero energy states. ${ }^{18}$

We thank Y. Morita for fruitful discussions. S.R. is grateful to T. Oka for useful comments. Y.H. was supported in part by a Grant-in-Aid from the Ministry of Education, Science, and Culture of Japan. The computation in this work was partly done at the YITP Computing Facility and at the Supercomputing Center, ISSP, University of Tokyo.
${ }^{1}$ R. Gade, Nucl. Phys. B 398, 499 (1993).

${ }^{2}$ T. Sugiyama and N. Nagaosa, Phys. Rev. Lett. 70, 1980 (1993); Y. Avishai, Y. Hatsugai, and M. Kohmoto, Phys. Rev. B 47, 9561 (1993).

${ }^{3}$ Y. Hatsugai, X.-G. Wen, and M. Kohmoto, Phys. Rev. B 56, 1061 (1997); Y. Morita and Y. Hatsugai, Phys. Rev. Lett. 79, 3728 (1997).

${ }^{4}$ C.-M. Ho and J.T. Chalker Phys. Rev. B 54, 8708 (1996).

${ }^{5}$ Y. Hatsugai and M. Kohmoto, Phys. Rev. B 42, 8282 (1990).

${ }^{6}$ P.A. Lee, Phys. Rev. Lett. 71, 1887 (1993); Y. Hatsugai and P.A. Lee, Phys. Rev. B 48, 4204 (1993).

${ }^{7}$ A.W.W. Ludwig, M.P.A. Fisher, R. Shankar, and G. Grinstein, Phys. Rev. B 50, 7526 (1994).

${ }^{8}$ Y. Aharonov and A. Casher, Phys. Rev. A 19, 2461 (1979).

${ }^{9}$ There is an analog of the Dirac equation [Eq. (1)] for chiral models on a lattice. Let $c_{A, B}$ represent the fermionic annihilation operator on sublattices $A$ and $B$, respectively. The chiral symmetry allows us to express the Hamiltonian of these models as

$$
\mathcal{H}=\left(\left\{c_{A}^{\dagger}\right\}\left\{c_{B}^{\dagger}\right\}\right)\left(\begin{array}{cc}
\mathbf{0}_{A} & \mathcal{D}_{A B} \\
\mathcal{D}_{A B}^{\dagger} & \mathbf{0}_{B}
\end{array}\right)\left(\begin{array}{l}
\left\{c_{A}\right\} \\
\left\{c_{B}\right\}
\end{array}\right) .
$$

Randomness resides in the off-diagonal parts $\mathcal{D}_{A B}$ and $\mathcal{D}_{A B}^{\dagger}$.
The zero-mode wave functions $\psi^{t}\left(\psi_{A}, \psi_{B}\right)$ satisfy the Schrödinger equations $\mathcal{D}_{A B} \psi_{B}=0$ and $\mathcal{D}_{A B}^{\dagger} \psi_{A}=0$.

${ }^{10}$ See, for example, B. Huckestein, Rev. Mod. Phys. 67, 357 (1995), and references therein.

${ }^{11}$ C. Chamon, C. Mudry and X.-G. Wen, Phys. Rev. Lett. 77, 4194 (1996).

${ }^{12}$ H.E. Castillo, C. Chamon, E. Fradkin, P.M. Goldbart, and C. Mudry, Phys. Rev. B 56, 10668 (1997).

${ }^{13}$ C. Mudry, C. Chamon, and X.-G. Wen, Nucl. Phys. B 466, 383 (1996).

${ }^{14}$ I.I. Kogan, C. Mudry, and A.M. Tsvelik, Phys. Rev. Lett. 77, 707 (1996).

${ }^{15}$ D. Carpentier and P. Le Doussal, cond-mat/0003281 (unpublished).

${ }^{16} \mathrm{~V}$. Gurarie, cond-mat/9907502 (unpublished).

${ }^{17}$ The RSB estimate for $\mathcal{F}_{n}$ was originally proposed for $0 \leqslant n<1$, and does not agree with the numerical replica estimate for $\mathcal{F}_{n=1,2,3,4,5}$. However, the success of the simple employment of the RSB estimate is mysterious, and seems to be related to the nature of the RSB. Further exploration would be an interesting future issue.

${ }^{18}$ S. Ryu and Y. Hatsugai (unpublished). 